\title{
Approximation by Sinc-Galerkin method for a class of fractional boundary value problems
}

\author{
Sebahat Ebru Das \\ Yildiz Technical University, Department of Mathematics, Istanbul, Turkey
}

Received: 18 October 2017, Accepted: 11 December 2017

Published online: 20 February 2018.

\begin{abstract}
The Fractional Boundary Value Problems (BVPs) oftenly come across in the modelling of several problems that we are faced in the branches of engineering sciences. The main aim of this work is to compute the solution of the Fractional BVP which is formed by $y^{\prime \prime}(x)+p(x) y^{\prime}(x)+q(x) y^{(\alpha)}(x)+r(x) y(x)=f(x), 0<\alpha<1$ with the boundary conditions $y(a)=0, y(b)=0$ approximately by using the Sinc-Galerkin Method. Conformable sense is considered for the fractional derivatives. Intercalarity, some test problems having exact solutions are approximately solved. Afterwards, the exact solutions compared with the approximate solutions and numerical results of that comparison are presented both in table and graphical forms.
\end{abstract}

Keywords: Fractional boundary value problem, Sinc-Galerkin method, conformable sense.

\section{Introduction}

Taking advantage of the fast and the prepotent processes with polynomials, the methods based on polynomials as Chebyshev, Legendre, Sinc, etc. would be rathered in a wide of engineering applications.Sinc methods are the methods as mentioned above with theirs helpful tool property. In fact, Sinc methods distinguish theirself from the other numerical methods with two main differences: exponential decrease in error and the reliability of the results come out at the singular points. The Sinc methods are applied to many engineering problems. For instance, in [1], the approximate solutions of several ordinary and partial differential equations in the plane are tackled by using Sinc-Galerkin Method. In [2], Troesch's problem is solved by using Sinc-Galerkin Method. In [3], a problem seen in the theory of chemical reactor is investigated approximately. In [4], Solution of the Bratu's problem appears in combustion theory of fuel ignition is presented. In [5], Biharmonic problems occur in several areas of mechanics are introduced and then solutions are given approximately.

We refer the interested reader to [6]-[15] for more information about the Sinc methods. In this work, we consider the following fractional BVP

$$
y^{\prime \prime}(x)+p(x) y^{\prime}(x)+q(x) y^{(\alpha)}(x)+r(x) y(x)=f(x) \quad 0<\alpha<1
$$

with the boundary conditions

$$
y(a)=0, y(b)=0
$$

where for $\alpha, y^{(\alpha)}$ is the comformable fractional derivative of $y(x)$. This paper is considered as follows. In section 2, The fundamental definitions and theorems are presented and the Sinc-Galerkin Method is identified. In section 3, The Sinc- 
Galerkin method is implemented to the equation (1) for the boundary conditions (2).In section 4, The comparison of the exact and the approximate solutions for certain test examples are presented both in tables and graphics. In section 5, the paper is completed with a conclusion.

\section{Preliminaries}

The basis definitions and theorems about fractional calculus and sinc functions are presented. For more details see [15-17].

Definition 1. Let $f:(0, \infty) \rightarrow R$ be a n-differentiable function. Then the conformable fractional derivative of order $\alpha$ for $n<\alpha \leq n+1$ off at $t$ is defined by

$$
T_{\alpha} f(t)=\lim _{\varepsilon \rightarrow 0} \frac{f^{(\lceil\alpha\rceil-1)}\left(t+\varepsilon t^{(\lceil\alpha\rceil-\alpha)}\right)-f^{(\lceil\alpha\rceil-1)}(t)}{\varepsilon}
$$

where $\lceil\alpha\rceil$ is the smallest integer greater than or equal to $\alpha$.

Remark.Let $f$ be a $(n+1)$-differentiable function at $\mathrm{t}$ and $n<\alpha \leq n+1$. As a result of Definition 1 ,

$$
T_{\alpha} f(t)=t^{(\lceil\alpha\rceil-\alpha)} f^{\lceil\alpha\rceil}(t)
$$

Theorem 1. Let $f$, $g$ be $\alpha$-differentiable functions at $t$ and $n<\alpha \leq n+1$.Then

(1) $T_{\alpha}(a f+b g)=a T_{\alpha}(f)+b T_{\alpha}(g)$, for all $a, b \in \mathbb{R}$.

(2) $T_{\alpha}\left(t^{p}\right)=p t^{p-\alpha}$, for all $p \in \mathbb{R}$

(3) $T_{\alpha}(\lambda)=0$, for all constant functions $f(t)=\lambda$.

(4) $T_{\alpha}(f g)=f T_{\alpha}(g)+g T_{\alpha}(f)$.

(5) $T_{\alpha}\left(\frac{f}{g}\right)=\frac{g T_{\alpha}(f)+f T_{\alpha}(g)}{g^{2}}$.

Definition 2. The function

$$
\operatorname{sinc}(x)= \begin{cases}\frac{\sin (\pi x)}{\pi x}, & x \neq 0 \\ 1, & x=0\end{cases}
$$

is called the Sinc (Sine Cardinal)function.

Definition 3. The translated sinc function with space point is defined by

$$
S(k, h)(x)=\operatorname{sinc}\left(\frac{x-k h}{h}\right)= \begin{cases}\frac{\sin \left(\pi \frac{x-k h}{h}\right)}{\pi \frac{x-k h}{h}} & x \neq k h \\ 1, & x=k h\end{cases}
$$

where $h>0$ and $k=0, \pm 1, \pm 2, \ldots$

Definition 4. The series which defined by

$$
C(f, h)(x)=\sum_{k=-\infty}^{\infty} f(k h) \operatorname{sinc}\left(\frac{x-k h}{h}\right)
$$

for $f(x)$ and $h>0$ is called the Whittaker Cardinal Expansion of $f$ whenever the series converges. For establishing the approximation on $(a, b)$, the conformable map is defined as

$$
\phi(z)=\ln \left(\frac{z-a}{b-z}\right)
$$


Here, the basis functions are attained using the composite translated sinc functions given as

$$
\begin{gathered}
S_{k}(z)=S(k, h)(z) o \phi(z)=\operatorname{sinc}\left(\frac{\phi(z)-k h}{h}\right) . \\
z=\phi^{-1}(w)=\frac{a+b e^{w}}{1+e^{w}}
\end{gathered}
$$

is the inverse map of $w=\phi(z)$. The sinc grid points $z_{k} \in(a, b)$ in $D_{E}$ are real numbers, so that they can denoted by $x_{k}$. For evenly spaced points $\{k h\}_{k=-\infty}^{\infty}$, the image corresponding to these points is defined by

$$
x_{k}=\phi^{-1}(k h)=\frac{a+b e^{k h}}{1+e^{k h}}, \quad k=0, \pm 1, \pm 2, \ldots
$$

Theorem 2. Let $F \in B\left(D_{E}\right)$ and $\Gamma$ be a real number on $(0,1)$. In that case, for small enough $h$,

$$
\int_{\Gamma} F(z) d z-h \sum_{j=-\infty}^{\infty} \frac{F\left(z_{j}\right)}{\phi^{\prime}\left(z_{j}\right)}=\frac{i}{2} \int_{\partial D} \frac{F(z) k(\phi, h)(z)}{\sin (\pi \phi(z) / h)} d z \equiv I_{F}
$$

where

$$
|k(\phi, h)|_{z \in \partial D}=\left|e^{\left[\frac{i \pi \phi(z)}{h} \operatorname{sgn}(\operatorname{Im} \phi(z))\right]}\right|_{z \in \partial D}=e^{\frac{-\pi d}{h}} .
$$

In sinc methods, the infinite quadrature should be cut with a finite sum.

Theorem 3. If there exist positive constants $\alpha, \beta$ and $C$ such that

$$
\left|\frac{F(x)}{\phi^{\prime}(x)}\right| \leq C\left\{\begin{array}{l}
e^{-\alpha|\phi(x)|}, x \in \psi((-\infty, \infty)) \\
e^{-\beta|\phi(x)|}, x \in \psi((0, \infty))
\end{array}\right.
$$

then the error limit for the quadrature rule (11) is

$$
\left|\int_{\Gamma} F(x) d x-h \sum_{j=-M}^{N} \frac{F\left(x_{j}\right)}{\phi^{\prime}\left(x_{j}\right)}\right| \leq C\left(\frac{e^{-\alpha M h}}{\alpha}+\frac{e^{-\beta N h}}{\beta}\right)+\left|I_{F}\right|
$$

The infinite sum in (11) is cut with the help of (13) to achieve (14). With the help of the choices

$$
h=\sqrt{\frac{\pi d}{\alpha M}} \text { and } N \equiv\left[\left\lfloor\frac{\alpha M}{\beta}+1\right\rfloor\right]
$$

knowing that $[\lfloor\rfloor$.$] is the integer part of the expression and M$ is the integer value that determines the size of the grid,

$$
\int_{\Gamma} F(x) d x=h \sum_{j=-M}^{N} \frac{F\left(x_{j}\right)}{\phi^{\prime}\left(x_{j}\right)}+O\left(e^{-(\pi \alpha d M)^{1 / 2}}\right) .
$$


Lemma 1. If $\phi$ is the conformal 1-1 mapping which simply connects the domain $D_{E}$ onto $D_{S}$. Then

$$
\begin{aligned}
& \delta_{j k}^{(0)}=\left.[S(j, h) o \phi(x)]\right|_{x=x_{k}} \begin{cases}1, & j=k \\
0, & j \neq k .\end{cases} \\
& \delta_{j k}^{(1)}=\left.h \frac{d}{d \phi}[S(j, h) o \phi(x)]\right|_{x=x_{k}} \begin{cases}0, & j=k \\
\frac{(-1)^{k-j}}{k-j}, & j \neq k .\end{cases} \\
& \delta_{j k}^{(2)}=\left.h^{2} \frac{d^{2}}{d \phi^{2}}[S(j, h) o \phi(x)]\right|_{x=x_{k}} \begin{cases}-\frac{\pi^{2}}{3}, & j=k \\
\frac{-2(-1)^{k-j}}{(k-j)^{2}}, & j \neq k .\end{cases}
\end{aligned}
$$

\section{The sinc-collocation method}

Consider the approximate solution of (1) is given by

$$
y_{m}(x)=\sum_{i=-M}^{N} c_{i} S_{i}(x), \quad m=M+N+1
$$

Here, $S_{i}$ is the composite function of $S(i, h)$ and $\phi(x)$ for some fixed step size $h$. The unknown coefficients $c_{i}$ in (18) are obtained with the help of orthogonalizing the residual with regard to the basis functions as

$$
\left\langle y^{\prime \prime}(x), S_{i}\right\rangle+\left\langle p(x) y^{\prime}(x), S_{i}\right\rangle+\left\langle q(x) y^{(\alpha)}(x), S_{i}\right\rangle+\left\langle r(x) y(x), S_{i}\right\rangle=\left\langle f(x), S_{i}\right\rangle, \quad i=-M, \ldots, N
$$

The inner product is defined by

$$
\langle f, g\rangle=\int_{a}^{b} f(x) g(x) w(x) d x
$$

In the inner product $w(x)$ is the weight function and it will be taken as

$$
w(x)=\frac{1}{\phi^{\prime}(x)}
$$

for the second order BVPs.

Theorem 4. The following relations are provided:

$$
\begin{aligned}
\left\langle y^{\prime \prime}(x), S_{i}\right\rangle & \approx h \sum_{j=-M}^{N} \sum_{k=0}^{2} \frac{y\left(x_{j}\right)}{\phi^{\prime}\left(x_{j}\right) h^{k}} \delta_{i j}^{(k)} g_{2, k}\left(x_{j}\right) \\
\left\langle p(x) y^{\prime}(x), S_{i}\right\rangle & \approx-h \sum_{j=-M}^{N} \sum_{k=0}^{1} \frac{y\left(x_{j}\right)}{\phi^{\prime}\left(x_{j}\right) h^{k}} \delta_{i j}^{(k)} g_{1, k}\left(x_{j}\right)
\end{aligned}
$$

If we take as $G(x)=r(x) y(x)$ and $G(x)=f(x)$, the inner product of $G(x)$ and $S_{i}$ will be

$$
\left\langle G, S_{i}\right\rangle \approx h \frac{G\left(x_{i}\right) w\left(x_{i}\right)}{\phi^{\prime}\left(x_{i}\right)}
$$

The proof is presented in [14].

Theorem 5. For $0<\alpha<1$, the following relation holds:

$$
\left\langle q(x) y^{(\alpha)}(x), S_{i}\right\rangle \approx-h \sum_{j=-M}^{N} \sum_{k=0}^{1} \frac{y\left(x_{j}\right)}{\phi^{\prime}\left(x_{j}\right) h^{k}} \delta_{i j}^{(k)} r_{1, k}\left(x_{j}\right)
$$


In the sum,

$$
\begin{aligned}
& r_{1,1}(x)=\left(q(x) x^{1-\alpha} w(x)\right) \phi^{\prime}(x) \\
& r_{1,0}(x)=\left(q(x) x^{1-\alpha} w(x)\right)^{\prime}
\end{aligned}
$$

Proof. The inner product of $q(x) y^{(\alpha)}(x)$ and $S_{i}$ is

$$
\begin{aligned}
\left\langle q(x) y^{(\alpha)}(x), S_{i}\right\rangle & =\int_{0}^{1} q(x) y^{(\alpha)}(x) S_{i}(x) w(x) d x \\
& =\int_{0}^{1} q(x) x^{1-\alpha} y^{\prime}(x) S_{i}(x) w(x) d x \\
& =\left.q(x) x^{1-\alpha} S_{i}(x) w(x) y(x)\right|_{0} ^{1}-\int_{0}^{1} y(x)\left(q(x) x^{1-\alpha} S_{i}(x) w(x)\right)^{\prime} d x
\end{aligned}
$$

With the use of the boundary conditions $y(0)=y(1)=0$, we write (26) as

$$
\left\langle q(x) y^{(\alpha)}(x), S_{i}\right\rangle=-\int_{0}^{1} y(x)\left(q(x) x^{1-\alpha} S_{i}(x) w(x)\right)^{\prime} d x
$$

So, we determine that

$$
\left\langle q(x) y^{(\alpha)}(x), S_{i}\right\rangle=-\int_{0}^{1} y(x)\left[S_{i}(x) r_{1,0}(x)+S_{i}^{(1)}(x) r_{1,1}(x)\right] d x
$$

where

$$
\begin{aligned}
& r_{1,1}(x)=\left(q(x) x^{1-\alpha} w(x)\right) \phi^{\prime}(x) \\
& r_{1,0}(x)=\left(q(x) x^{1-\alpha} w(x)\right)^{\prime}
\end{aligned}
$$

Using (16) and lemma 1 , it is shown that

$$
\begin{aligned}
\left\langle q(x) y^{(\alpha)}(x), S_{i}\right\rangle & \approx-h \sum_{j=-M}^{N} \frac{y\left(x_{j}\right)}{\phi^{\prime}\left(x_{j}\right)}\left[\frac{1}{h} \delta_{i j}^{(1)} r_{1,1}\left(x_{j}\right)+\delta_{i j}^{(0)} r_{1,0}\left(x_{j}\right)\right] \\
& =-h \sum_{j=-M}^{N} \sum_{k=0}^{1} \frac{y\left(x_{j}\right)}{\phi^{\prime}\left(x_{j}\right) h^{k}} \delta_{i j}^{(k)} r_{1, k}\left(x_{j}\right)
\end{aligned}
$$

Replacing each term of (19) with the approach defined in (22)-(25), $y\left(x_{j}\right)$ with $c_{j}$, and dividing by $h$, we state the following theorem.

Theorem 6. Let us consider the boundary value problem (1)-(2).Then then the discrete Sing-Galerkin system for the approximate solution If the assumed approximate solution of the boundary-value problem (1) is (2), then the discrete SincGalerkin system for obtain the unknown coefficients $\left\{c_{j}\right\}_{j=-M}^{N}$ for the approximate solution is given, for $i=-M, \ldots, N$,

$$
\sum_{j=-M}^{N}\left\{\sum_{k=0}^{2} \frac{1}{h^{k}} \delta_{i j}^{(k)} \frac{g_{2, k}\left(x_{j}\right)}{\phi^{\prime}\left(x_{j}\right)} c_{j}-\sum_{k=0}^{1} \frac{1}{h^{k}} \delta_{i j}^{(k)} \frac{g_{1, k}\left(x_{j}\right)}{\phi^{\prime}\left(x_{j}\right)} c_{j}-\sum_{k=0}^{1} \frac{1}{h^{k}} \delta_{i j}^{(k)} \frac{r_{1, k}\left(x_{j}\right)}{\phi^{\prime}\left(x_{j}\right)} c_{j}+\frac{r\left(x_{i}\right) w\left(x_{i}\right)}{\phi^{\prime}\left(x_{i}\right)} c_{i}\right\}=\frac{f\left(x_{i}\right) w\left(x_{i}\right)}{\phi^{\prime}\left(x_{i}\right)}
$$

Some notations are defined to represent the system (28) in the matrix-vector form. Let $\mathbf{D}(y)$ be a diagonal matrix whose diagonal elements are $y\left(x_{-M}\right), y\left(x_{-M+1}\right), \ldots, y\left(x_{N}\right)$ and non-diagonal elements are zero. In addition, let $\mathbf{I}^{(k)}$ denotes the 
matrices for $0 \leq k \leq 2$ by

$$
\mathbf{I}^{(k)}=\left[\delta_{j i}^{(k)}\right], \quad j, i=-M, \ldots, N
$$

where $\mathbf{D}, \mathbf{I}^{(0)}, \mathbf{I}^{(1)}$ and $\mathbf{I}^{(2)}$ are square matrices of order $n \times n$. By using the above notations in the system (28), we can rewrite this system as

$$
\mathbf{A c}=\mathbf{B}
$$

where

$$
\begin{aligned}
\mathbf{A} & =\sum_{j=0}^{2} \frac{1}{h^{j}} \mathbf{I}^{(2)} \mathbf{D}\left(\frac{g_{2, j}}{\phi^{\prime}}\right)-\sum_{j=0}^{1} \frac{1}{h^{j}} \mathbf{I}^{(1)} \mathbf{D}\left(\frac{g_{1, j}}{\phi^{\prime}}\right)-\sum_{j=0}^{1} \frac{1}{h^{j}} \mathbf{I}^{(1)} \mathbf{D}\left(\frac{r_{1, j}}{\phi^{\prime}}\right)+\mathbf{I}^{(0)} \mathbf{D}\left(\frac{g_{0,0}}{\phi^{\prime}}\right) \\
\mathbf{B} & =\mathbf{D}\left(\frac{w f}{\phi^{\prime}}\right) \mathbf{1} \\
\mathbf{c} & =\left(c_{-M}, c_{-M+1}, \ldots, c_{N-1}, c_{N}\right)^{T}
\end{aligned}
$$

Finally, we can reach the approximate solution of (28) after finding the unknown coefficients $c_{i}$ in the system.

\section{Numerical examples}

In this section, HWM is applied to some test examples using MATHEMATICA 10. For the all examples, we consider $h=\pi / \sqrt{N}$ and $N=M=L$.

Example 1. Let us take the linear fractional BVP

$$
y^{\prime \prime}(x)+y^{(0.3)}(x)+y(x)=x^{2}\left(x^{3}+5 x^{2.7}-x^{2}-4 x^{1.7}+20 x-12\right)
$$

with the boundary conditions

$$
y(0)=0, y(1)=0
$$

This BVP has an exact solution in the form of $y(x)=x^{4}(x-1)$. The approximate solution obtained with the aid of HWM of this problem is shown in table 1. In addition, the exact and the approximate solutions of the problem are shown graphically for different $\mathrm{n}$ values in figure 1 .

Example 2. Let us assume the following fractional BVP

$$
y^{\prime \prime}(x)+\cos (x) y^{\prime}(x)-x y^{(0.7)}(x)=-\cos ^{2}(x)(x-1)+\sin (x)\left(x^{1.3}+x-1\right)-\cos (x)\left(\sin (x)-x^{2.3}+x^{1.3}+2\right)
$$

adhere to the boundary conditions

$$
y(0)=0, y(1)=0
$$

The exact solution of the problem is $y(x)=\sin (x)(1-x)$. The numerical solutions determined by HWM of the problem are presented in Table 2. Furthermore, the comparisons of the exact and the approximate solutions for different values of $N$ are shown in Figure 2. 
Table 1: Absolute errors for Example 1 for different values of $N$.

\begin{tabular}{clllll}
\hline$x$ & $N=4$ & $N=8$ & $N=16$ & $N=32$ & $N=64$ \\
\hline \hline 0.1 & $3.642 \times 10^{-3}$ & $8.128 \times 10^{-4}$ & $4.833 \times 10^{-5}$ & $7.310 \times 10^{-7}$ & $1.566 \times 10^{-9}$ \\
0.2 & $4.766 \times 10^{-3}$ & $3.613 \times 10^{-4}$ & $7.901 \times 10^{-5}$ & $9.341 \times 10^{-7}$ & $1.896 \times 10^{-9}$ \\
0.3 & $7.281 \times 10^{-3}$ & $9.350 \times 10^{-4}$ & $1.808 \times 10^{-5}$ & $1.207 \times 10^{-6}$ & $7.897 \times 10^{-10}$ \\
0.4 & $6.540 \times 10^{-3}$ & $7.322 \times 10^{-4}$ & $6.473 \times 10^{-5}$ & $1.359 \times 10^{-6}$ & $1.880 \times 10^{-9}$ \\
0.5 & $5.856 \times 10^{-3}$ & $2.405 \times 10^{-5}$ & $3.038 \times 10^{-5}$ & $8.176 \times 10^{-7}$ & $2.318 \times 10^{-9}$ \\
0.6 & $6.228 \times 10^{-3}$ & $1.694 \times 10^{-4}$ & $4.361 \times 10^{-5}$ & $2.879 \times 10^{-7}$ & $2.366 \times 10^{-9}$ \\
0.7 & $5.953 \times 10^{-3}$ & $1.543 \times 10^{-5}$ & $2.370 \times 10^{-5}$ & $7.500 \times 10^{-7}$ & $2.965 \times 10^{-9}$ \\
0.8 & $1.917 \times 10^{-3}$ & $2.463 \times 10^{-4}$ & $3.685 \times 10^{-6}$ & $5.934 \times 10^{-7}$ & $1.516 \times 10^{-9}$ \\
0.9 & $3.166 \times 10^{-3}$ & $3.296 \times 10^{-4}$ & $1.574 \times 10^{-5}$ & $3.348 \times 10^{-7}$ & $7.106 \times 10^{-10}$ \\
\hline
\end{tabular}

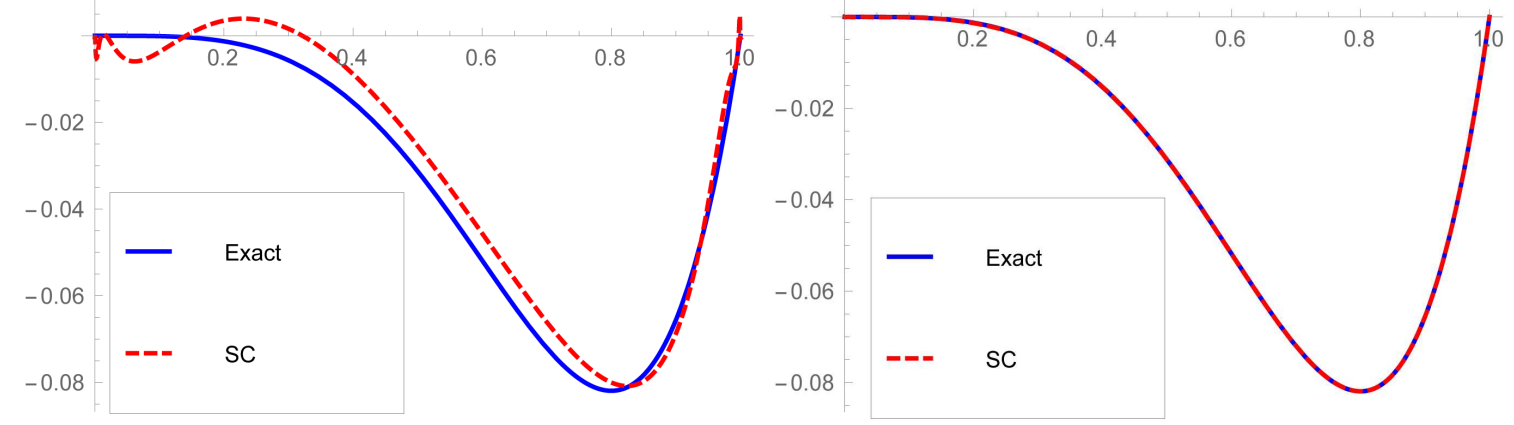

(a) $N=4$

(b) $N=16$

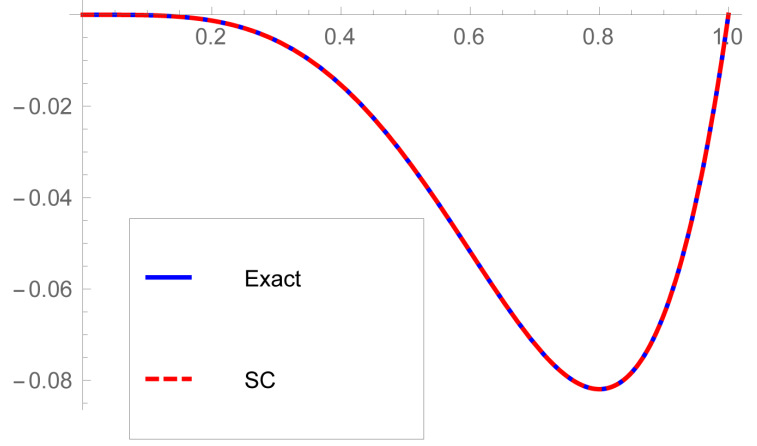

(c) $N=64$

Fig. 1: Graphs of exact and approximate solutions for Example 1.

\section{Competing interests}

The authors declare that they have no competing interests.

\section{Authors' contributions}

All authors have contributed to all parts of the article. All authors read and approved the final manuscript. 
Table 2: Absolute errors for Example 2 for different values of $N$.

\begin{tabular}{llllll}
\hline$x$ & $N=4$ & $N=8$ & $N=16$ & $N=32$ & $N=64$ \\
\hline \hline 0.1 & $4.292 \times 10^{-3}$ & $6.072 \times 10^{-4}$ & $1.513 \times 10^{-5}$ & $1.321 \times 10^{-7}$ & $8.661 \times 10^{-10}$ \\
0.2 & $3.582 \times 10^{-3}$ & $3.610 \times 10^{-4}$ & $2.868 \times 10^{-5}$ & $3.814 \times 10^{-7}$ & $8.919 \times 10^{-10}$ \\
0.3 & $4.518 \times 10^{-3}$ & $3.692 \times 10^{-4}$ & $1.479 \times 10^{-5}$ & $4.035 \times 10^{-7}$ & $8.881 \times 10^{-10}$ \\
0.4 & $2.644 \times 10^{-3}$ & $3.849 \times 10^{-4}$ & $1.962 \times 10^{-5}$ & $3.031 \times 10^{-8}$ & $8.497 \times 10^{-10}$ \\
0.5 & $1.367 \times 10^{-3}$ & $1.823 \times 10^{-4}$ & $1.690 \times 10^{-5}$ & $3.697 \times 10^{-7}$ & $9.330 \times 10^{-10}$ \\
0.6 & $1.701 \times 10^{-3}$ & $1.032 \times 10^{-4}$ & $3.800 \times 10^{-6}$ & $3.052 \times 10^{-7}$ & $9.113 \times 10^{-10}$ \\
0.7 & $2.620 \times 10^{-3}$ & $1.803 \times 10^{-6}$ & $1.908 \times 10^{-5}$ & $2.509 \times 10^{-8}$ & $9.424 \times 10^{-10}$ \\
0.8 & $1.632 \times 10^{-3}$ & $2.900 \times 10^{-4}$ & $6.602 \times 10^{-6}$ & $2.087 \times 10^{-7}$ & $6.082 \times 10^{-11}$ \\
0.9 & $2.410 \times 10^{-3}$ & $8.289 \times 10^{-5}$ & $4.529 \times 10^{-6}$ & $3.354 \times 10^{-7}$ & $5.932 \times 10^{-11}$ \\
\hline
\end{tabular}

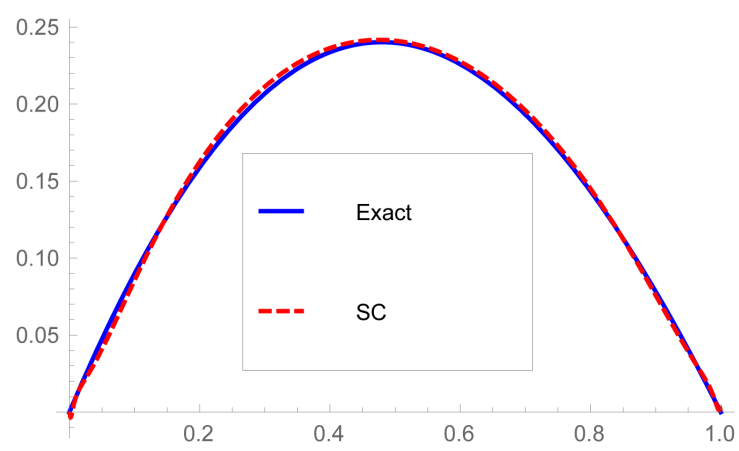

(a) $N=4$

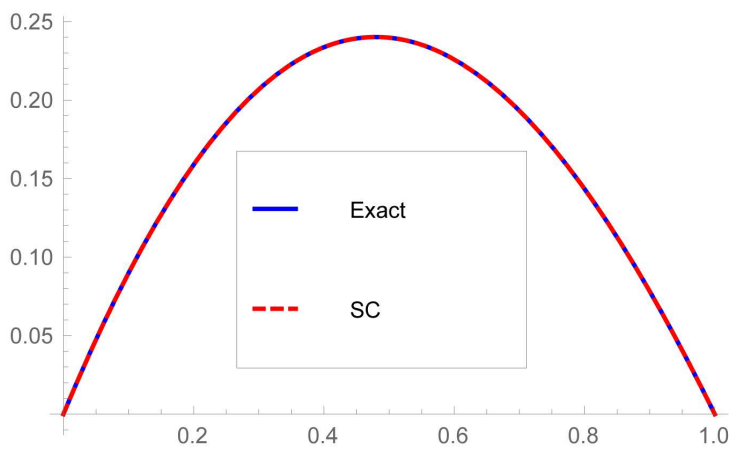

(b) $N=16$

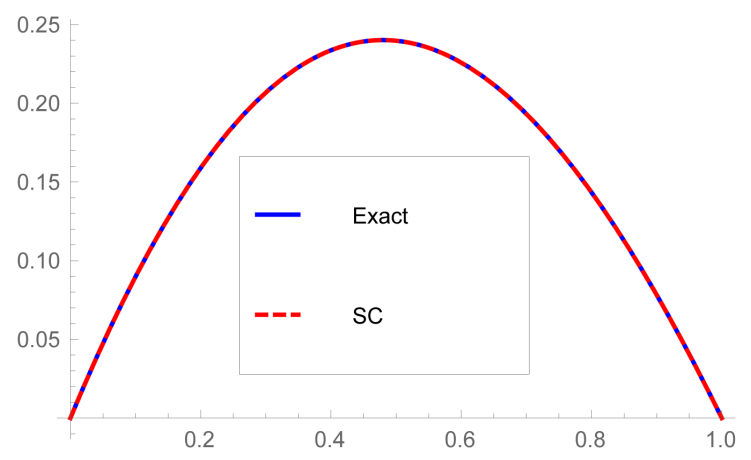

(c) $N=64$

Fig. 2: Graphs of exact and approximate solutions for Example 2.

\section{Conclusion}

The Sinc-Galerkin Method is applied to the different fractional order differential equations. All computations associated with the examples are done using Mathematica. The exact and approximate solutions are compared for all the examples. It can be concluded that the Sinc-Galerkin method is a quite effective and accurate method. We aim to apply the SincGalerkin Method to the fractional order partial differential equations in our later studies. 


\section{References}

[1] J. Lund, K. Bowers, "Sinc Methods for Quadrature and Differential Equations", SIAM, Philadelphia, 1992.

[2] F. Stenger, "Numerical Methods Based on Sine and Analytic Functions", Springer-Verlag, New York, 1993.

[3] F. Stenger, "A Sinc-Galerkin Method of Solution of Boundary Value Problems," Mathematics of Computation, vol. 33(145), pp. 85-109, 1979.

[4] M. Zarebnia and M. Sajjadian, "Sinc-Galerkin Method for Solving Biharmonic Problems" Mathematical and Computer Modelling, Vol.56(9-10), pp. 218-228, 2012.

[5] A. Saadatmandi, M. Razzaghi and M. Dehghan, ”Sinc-Galerkin Solution for Nonlinear Two-point Boundary Value Problems with Applications to Chemical Reactor Theory", Mathematical and Computer Modelling, Vol.42(11-12), pp.1237-1244, 2005.

[6] Jalil Rashidinia, Khosro Maleknejad and Narges Taheri, "Sinc-Galerkin Method for Numerical Solution of the Bratu's Problems", Numerical Algoritms, Vol. 62(1), pp.1-11, 2013.

[7] Mohamed El-Gamel, Adel Mohsen and Amgad Abd El-Mohsen, "Sinc-Galerkin Method for Solving Biharmonic Problems", Applied Mathematics and Computation, Vol.247(15), pp.386-396, 2014.

[8] S. Alkan and A. Secer, "Application of Sinc-Galerkin Method for Solving Space-Fractional Boundary Value Problems", Mathematical Problems in Engineering, Art. ID. 217348, 10 pages, 2015.

[9] K. Abdella, X.Yu and I. Kucuk, ”Application of the Sinc Method to a Dynamic Elasto-Plastic Problem”, Journal of Computational and Applied Mathematics, Vol. 223, pp.626-645, 2009.

[10] A. Secer, "Numerical Solution and Simulation of Second-Order Parabolic PDEs with Sinc-Galerkin Method Using Maple", Abstract and Applied Analysis, Art. ID. 686483, 10 pages, 2013.

[11] A. Zakeri, A. H. S. Shayegan and F. Asadollahi, ”A Numerical Method for Solving Nonlinear Partial Differential Equations Based on Sinc-Galerkin Method”, Journal of Mathematical Modelling, Vol5(1), pp. 27-40, 2017.

[12] E. Babolian, A. Eftekhari and A. Saadatmandi, ”A Sinc-Galerkin Approximate Solution of the Reaction-Diffusion Process in an Immobilized Biocatalyst Pellet", Communications in Mathematical and in Computer Chemistry, Vo71, pp. 681-697, 2014.

[13] M. A. Hajji and K. Al-Khaled, "Numerical Methods for Nonlinear Fourth-Order Bonudary Value Problems with Applications", Vol. 85(1), pp.83-104, 2008.

[14] A. Secer, S. Alkan, M.A. Akinlar and M. Bayram, "Sinc-Galerkin Method for Approximate Solutions of Fractional Order Boundary Value Problems", Boundary Value Problems, Art. ID. 2013:281, 2013.

[15] A. Shidfar and A. Babaei, "The Sinc-Galerkin Method for Solving an Inverse Parabolic Problem with Unknown Source Term", Numerical Methods for Partial Differential Equations, Vol. 29(1), pp.64-78, 2013.

[16] M. Dehghan and F. Emami-Naeini, "The Sinc-collocation and Sinc-Galerkin methods for solving the two-dimensional Schrdinger equation with nonhomogeneous boundary conditions", Applied Mathematical Modelling, Vol.37, pp.9379-9397, 2013.

[17] Alkan, S., Hatipoglu, V. F., "Approximate solutions of Volterra-Fredholm integro-differential equations of fractional order", Tbilisi Mathematical Journal, Vol:10, Issue:2, pp: 1-13, 2017. 